\title{
MHD SIMULATION OF CHROMOSPHERIC EVAPORATION IN A SOLAR FLARE BASED ON MAGNETIC RECONNECTION MODEL
}

\author{
T. YOKOYAMA AND K. SHIBATA \\ National Astronomical Observatory of Japan \\ Mitaka, Tokyo, 181 Japan
}

Two-dimensional magnetohydrodynamic simulation of a solar flare is performed using a newly developed MHD code including nonlinear anisotropic heat conduction effect (Fig. 1; Yokoyama \& Shibata 1997a). The numerical simulation starts with a vertical current sheet which is line-tied at one end to a dense chromosphere. The flare energy is released by the magnetic reconnection mechanism stimulated initially by the resistivity perturbation in the corona. The released thermal energy is transported in to the chromosphere by heat conduction and drives chromospheric evaporation. Owing to the heat conduction effect, the adiabatic slow-mode MHD shocks emanated from the neutral point are dissociated into conduction fronts and isothermal shocks (Yokoyama \& Shibata 1997b). Temperature and derived soft $\mathrm{X}$-ray distributions are similar to the cusp-like structure of long-durationevent (LDE) flares observed by the soft X-ray telescope aboard Yohkoh satellite. On the other hand density and radio maps show a simple loop configuration which is consistent with the observation with Nobeyama Radio Heliograph. Two interesting new features are found. One is a pair of high density humps on the evaporated plasma loops formed at the collision site between the reconnection jet and the evaporation flow. The other is the loop-top blob behind the fast-mode MHD shock.

\section{References}

Yokoyama, T. and Shibata K. (1997a) Two-Dimensional Magnetohydrodynamic Simulation of Chromospheric Evaporation in Solar Flare Based on Magnetic Reconnection Model, submitted to ApJ Letters.

Yokoyama, T. and Shibata K. (1997b) Magnetic Reconnection Coupled with Heat Conduction, Astrophys. J., 474, L61. 

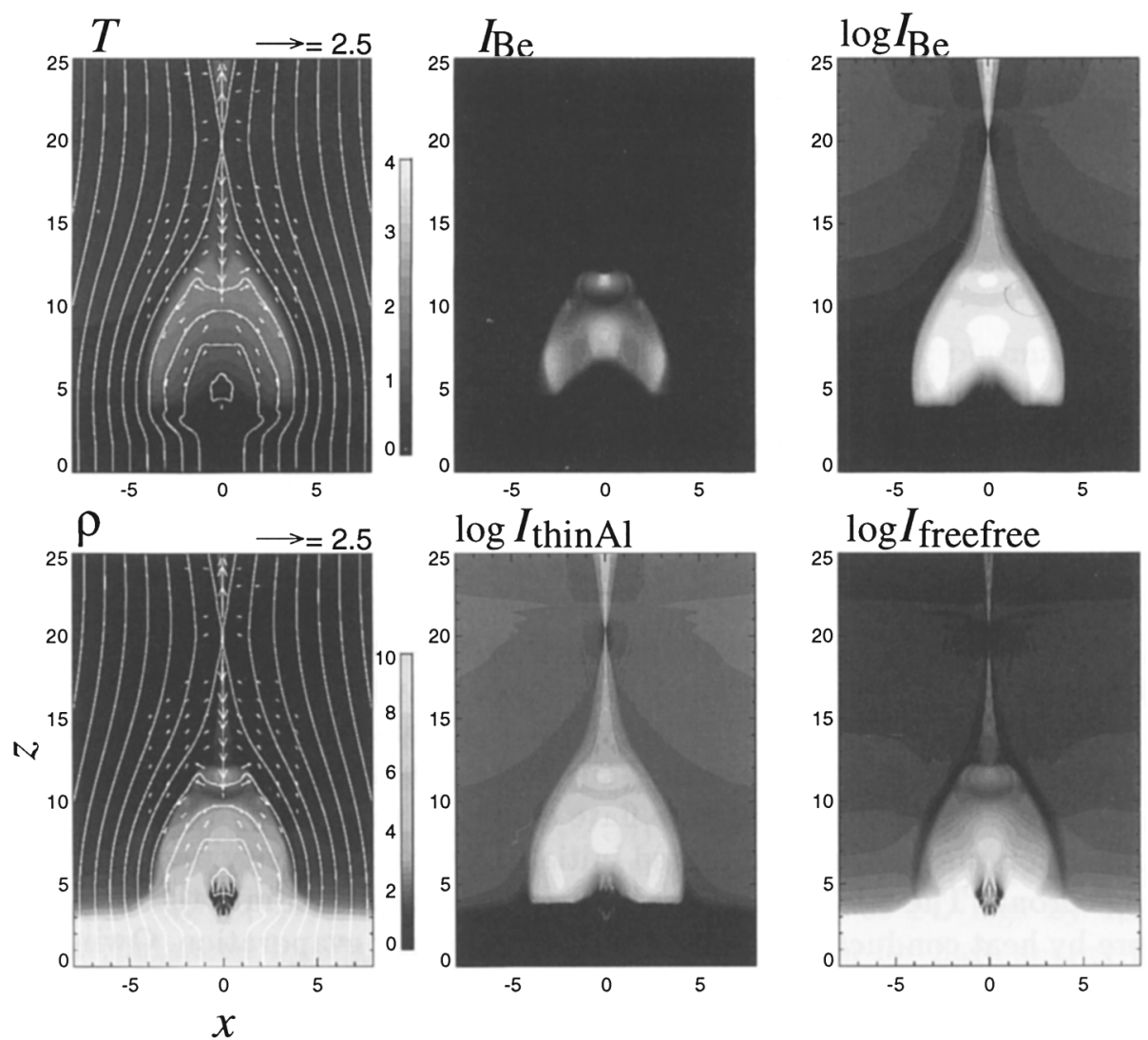

Figure 1. Results of the simulation. Snapshots at $t=25$ of temperature, density, $\mathrm{X}$-ray and radio maps derived from the simulation results are shown. The arrows show the velocity, and lines show the magnetic field lines. The unit of length, velocity, time, temperature, and density is $3000 \mathrm{~km}, 170 \mathrm{~km} \mathrm{~s}{ }^{-1}, 18 \mathrm{~s}, 2 \times 10^{6} \mathrm{~K}$, and $10^{9} \mathrm{~cm}^{-3}$, respectively. For the $\mathrm{X}$-ray map, the response of the filter attached to the soft $\mathrm{X}$-ray telescope on board Yohkoh is taken into account. $I_{\mathrm{Be}}$ and $I_{\mathrm{thinAl}}$ are the intensity through the beryllium and the thin aluminum filters. The radio map $I_{\text {freefree }}$ is derived under the assumption that the emission is by the thermal free-free mechanism. The unit of intensity is arbitrary. 